\title{
Taming the Janssen effect
}

\author{
Louison Thorens ${ }^{1,2, *}$, Knut Jørgen Måløy ${ }^{2}$, Mickaël Bourgoin ${ }^{1}$, and Stéphane Santucci1,3 \\ ${ }^{1}$ Univ Lyon, ENS de Lyon, Univ Claude Bernard, CNRS, Laboratoire de Physique, F-69342 Lyon, France \\ ${ }^{2}$ PoreLab, The Njord Centre, Department of Physics, University of Oslo, P. O. Box 1048 Blindern, N-0316 Oslo, Norway \\ ${ }^{3}$ Lavrentyev Institute of Hydrodynamics, Siberian Branch of the Russian Academy of Sciences, Novosibirsk, Russia
}

\begin{abstract}
We investigate both experimentally and theoretically the apparent mass of a ferromagnetic granular assembly filling a cylindrical container and submitted to a magnetic field $\mathbf{B}$, aligned vertically along the silo. We show that the mass of the ferromagnetic granular column depends strongly on the applied magnetic field. Notably, our measurements deviate strongly from the exponential saturation of the measured mass as a function of the true mass of the grain packing, as predicted by Janssen [H.A. Janssen, Vereins Eutscher Ingenieure Zeitschrift, 1045 (1895)]. In particular, the measured mass of tall columns decreases systematically as the amplitude of the magnetic field increases. We rationalize our experimental findings by considering the induced magnetic dipole-dipole interactions within the whole packing. We show the emergence of a global magnetic radial force along the walls of the silos, fully determined by the external magnetic field. The resulting tunable frictional interactions allows a full control of the effective mass of the ferromagnetic granular column.
\end{abstract}

\section{Introduction}

More than hundred years ago, a German engineer H.A Janssen studying the failure of silos, proposed a phenomenological model to describe the saturation of the pressure measured at the bottom of a container filled with grains [1]. Indeed, the pressure exerted at the bottom of a silo does not follow the hydrostatic formula $\sigma=\rho g h$ where $\rho$ is the average density of the medium and $h$ the column height, but saturates. Janssen's approach of this problem is simple but transcript nicely the experimental measurements. It can be resumed as follow: (i) the grain assembly is considered as a continuous medium with a homogeneous vertical stress $\sigma_{z}$ along a tube section, (ii) within the grains packing, the vertical stress is redistributed in the horizontal direction following the proportional law: $\sigma_{r}=K \sigma_{z}$, with a phenomenological constant $K$, (iii) the frictional contact forces $F_{z}$ between the grains and the cylinder wall are at the Coulomb limit $F_{z}=\mu F_{r}$, with $\mu$ the friction coefficient. Using these three hypothesis on a column slice, Janssen could demonstrate that the apparent mass measured at the base of the silo saturates exponentially, $m_{J}=m_{\infty}\left[1-\exp \left(-m_{0} / m_{\infty}\right)\right]$ characterized by the saturation mass $m_{\infty}=\left(\rho \pi R^{3}\right) /(2 K \mu)$, with $R$ the tube radius. During the last decades this simple approach has been criticized and refined [2-7], but it remains a classical result nowadays, the so-called, Janssen effect. In fact, when dealing with this static configuration of grains at rest in a tube, the main issue resides in the measure of the stresses since a granular system at rest is extremely responsive to perturbation and its preparation [8]. To measure the apparent mass at the bottom of the system, [9] pro-

*e-mail: louison.thorens@ens-lyon.fr

A video is available at https://doi.org/10.48448/2292-nz67 posed an experimental protocol to ensure that the friction forces are fully mobilized in the vertical direction. Nevertheless the Janssen effect is ultimately hardly controllable. Here, we propose to use ferromagnetic grains which, when submitted to an external magnetic field, acquire a magnetic moment leading to tunable pair interactions inside the granular medium. The possibility to modify and control in-situ with an external parameter the particles interactions within the packing constitutes a great advantage, in stark contrast with other means, such as the use of different particles (with different shapes and roughness) [10, 11], modifying the environment humidity (leading to capillary bridges) or triboelectric charge effects [12-14], which are all barely tunable.

\section{Experiments}

A sketch of our experimental setup is shown in figure 1a. This setup is composed of a copper tube of $19.6 \mathrm{~mm}$ radius filled with monodisperse $0.75 \mathrm{~mm}$ radius steel grains laying on a $19.0 \mathrm{~mm}$ radius piston fixed to a force gauge. The sensor is also mounted on a motorized vertical translation stage moving downward. The whole setup is placed between two coils mounted in a Helmholtz configuration, generating a uniform magnetic field $\mathbf{B}$ in the vertical direction as shown in figure $1 b$.

To quantify the effect of the magnetic interaction, we can consider the dimensionless number $\Psi$, (sometimes referred as a magnetic Bond number $[15,16]$ ), which compares magnetic pair interaction and gravity, $\Psi=$ $\left(\chi_{m} B\right)^{2} /\left(\mu_{0} a \rho g\right)$ where $\chi_{m}$ is the magnetic susceptibility of the beads equal to 3 in the case of a random close packing [17], $\mu_{0}$ is the magnetic permeability of vacuum, $a$ the 
(a)

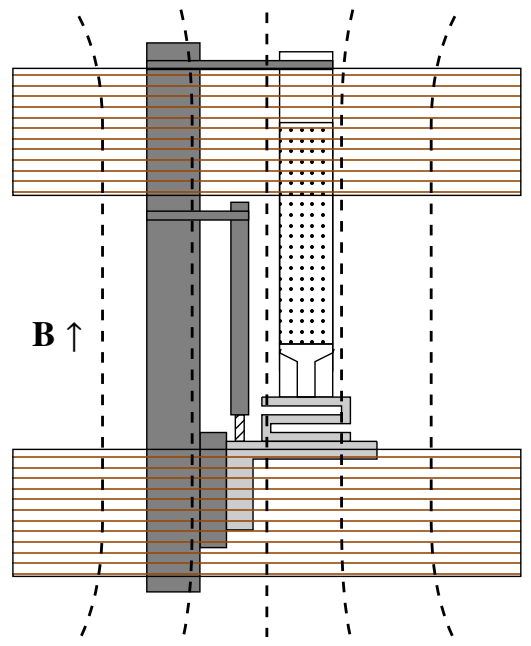

(b)

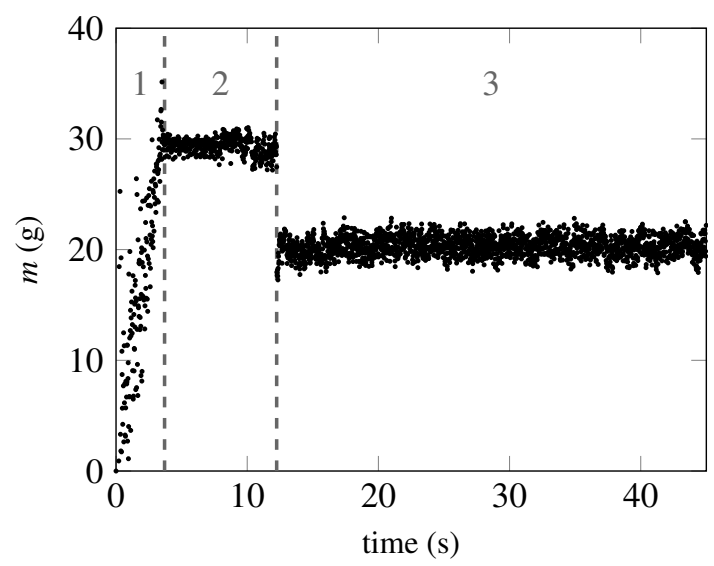

Figure 1. (a) Sketch of the experimental set-up with the generation of a homogeneous vertical magnetic field, using coils in a Helmholtz configuration. (b) Typical mass measurement, 1. beads are poured inside a tube using a hopper until we reach the desired mass $\mathrm{m} 0,2$. the packing is at rest and the hopper is removed, 3. downward movement of the vertical translation stage, the mass measurement corresponds to the mean plateau value.

bead radius, $\rho$ the bead density. In our case, due to the limitation of the power supply used, the maximum reachable value is $\Psi=35$ corresponding to a magnetic field $B=170 \mathrm{G}$.

Figure $1 \mathrm{~b}$ gives a typical mass measurement using our system. Our protocol is the following: the grains are poured inside the tube using a funnel until reaching the desired packing mass $m_{0}$. Then the funnel is removed and the magnetic field turned on. The vertical translation stage moves downward at a constant velocity $v=0.2 \mathrm{~mm} \mathrm{~s}^{-1}$ over a distance of $\Delta h \simeq 7 \mathrm{~cm}$, following the protocol proposed by [9], releasing the spring compression loaded in the mass sensor, leading to a lower apparent mass. The mass reaches a plateau during the descent where the mean value corresponds to our mass measurement $m$ and the standard deviation gives the typical dispersion of our measurement. Finally, when the stage stops, the mass sensor is compressed again and the apparent mass is slightly higher than the mean plateau value.

We used this experimental protocol to measure the apparent mass of the grain column for different packing mass $m_{0}$ and field amplitude $\Psi$. The results are shown in figure 2a. First, without magnetic field, $\Psi=0$, the measured mass of the granular column is in very good agreement with Janssen's prediction, following an exponential saturation with a characteristic mass $m_{\infty}=42 \mathrm{~g}$. Nevertheless, as soon as the ferromagnetic granular column is submitted to an external magnetic field, we observe a strong deviation from Janssen's prediction. Specifically, when increasing the amplitude of the magnetic field, we clearly see that for a high enough packing mass $m_{0}>40 \mathrm{~g}$, the higher the magnetic field, the lower the apparent mass. The even more striking effect is clearly seen for the highest amplitude of the magnetic field used, corresponding to $\Psi=35$, for which from a certain packing mass $m_{0} \approx 70 \mathrm{~g}$, the apparent mass of the packing decreases with the actual mass of the packing $m_{0}$ : the more added grains, the lower the apparent mass! Pushing the limits of our experimental setup, using a very tall column corresponding to a mass $m 0=190 \mathrm{~g}$ where the magnetic field is not as homogeneous, we could measure that the apparent mass became null for the highest amplitude of the magnetic field applied. Indeed, in such conditions, the grain assembly was stuck inside the tube, its wall supporting completely the weight of the granular column. To emphasize such deviations to the prediction of Janssen, we show on figure $2 b$ the difference between the apparent mass $m$ and the Janssen classical prediction $m_{J}$ shown in figure $2 \mathrm{~b}$. For low packing mass $m_{0}<40 \mathrm{~g}$, the apparent mass is slightly higher than the Janssen prediction, while for higher packing mass the measure is always lower than the classical prediction and seems to follow a linear slope $\left(m_{0}>40 \mathrm{~g}\right)$ which decreases systematically with the magnetic field amplitude.

\section{Model}

Ferromagnetic grains under a uniform vertical magnetic field acquire a vertical magnetic moment $\mathbf{d}$ directly proportional to the magnetic field via the magnetic susceptibility, $\mathbf{d}=V \chi_{m} \mathbf{B} / \mu_{0}$. Each particle $i$ of the packing will interact with a particle $\mathrm{j}$ with the following magnetic dipoledipole interactions [18]:

$$
\mathbf{f}_{\mathbf{i j}}=\frac{3 \mu_{0}}{4 \pi r_{i j}^{5}}\left(d^{2}\left(1-5 \cos ^{2} \alpha\right) \mathbf{r}_{\mathbf{i j}}+2 d r_{i j} \mathbf{d} \cos \alpha\right),
$$

where $\alpha$ is the angle between the magnetic field and the beads distance vector $\mathbf{r}_{i j}$. Figure 3 a gives a representation of the magnetic interaction either repulsive or attractive depending on the relative position of the particles $i$ and $j$ $\left(\mathbf{r}_{\mathbf{i j}}\right)$, and the applied magnetic field $\mathbf{B}$, characterized by the angle $\alpha$. Therefore, a particle $i$ of the packing will be subjected to multiple interactions from its neighbours following each time the expression above. The resulting magnetic force will be the sum of all magnetic dipole-dipole interactions $f_{i}=\sum_{j} f_{i j}$.

We can first discuss those local magnetic forces $f_{i}$, within the granular packing. Because of the cylindrical symmetry 
(a)

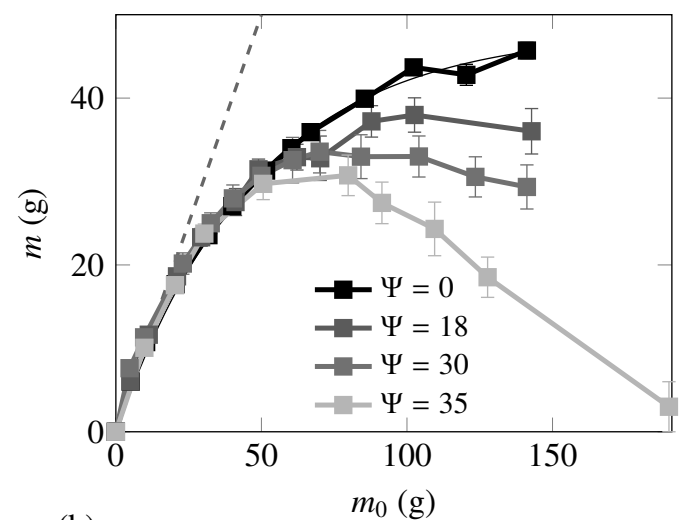

(b)

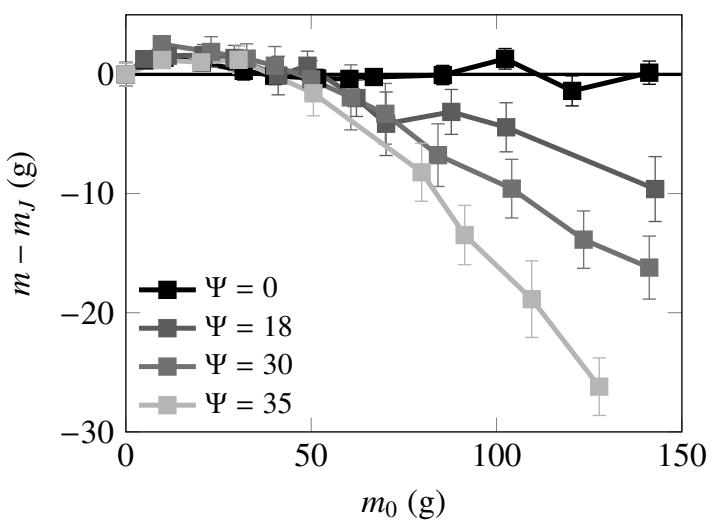

Figure 2. (a) Apparent mass measured using our experimental setup for different magnetic field amplitude, the black continuous line corresponds to the Janssen model $m_{J}$ and the grey dashed line represents the hydrostatic limit. (b) Mass difference between the apparent mass of the system and the Janssen expression $m_{J}$ for different magnetic field amplitude.

of the column, and the mirror symmetry with respect to the mid-plane at $z=h / 2$, the magnetic interactions do not add any global azimuthal nor vertical forces on the packing. Thus, the only net force that will not average to zero within the column, is the radial component $f^{r}$. The figure $3 \mathrm{~b}$ describes schematically this effect, which arises because of the finite-size of the silo, and the non-compensation of some interactions due to missing particles (displayed in white). It is important to remark also that we can expect this effect to be maximal along the walls of the container. To verify these considerations, we generated 3D random close packing using Discrete Element Method simulations [19]. We followed the numerical protocol the filling protocol proposed by [20] where the particles are randomly placed on a disk at constant height above the sedimented packing. This simulation method allowed us to generate packings of filling fraction $\phi \approx 0.6$, as shown in figure $3 \mathrm{c}$. Using the positions of the beads thus generated, we computed all the magnetic pair interactions using eq. (1). Thus, we could obtain the magnetic force exerted on each particle inside the packing. We could obtain the magnetic force exerted for a packing vertical slice in figure $3 \mathrm{~d}$, with vectors in red (resp. blue) for a positive (resp. negative) (a)

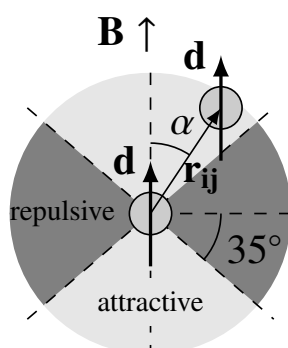

(c)

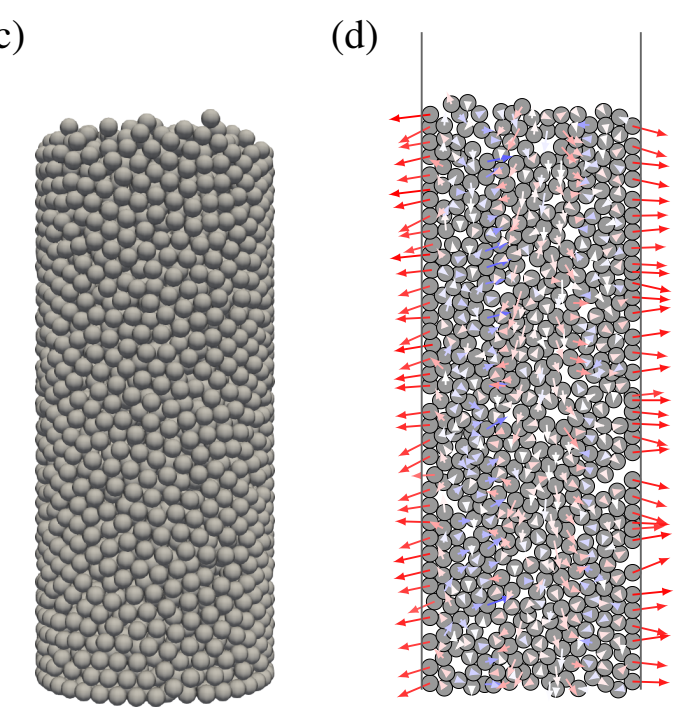

(b)

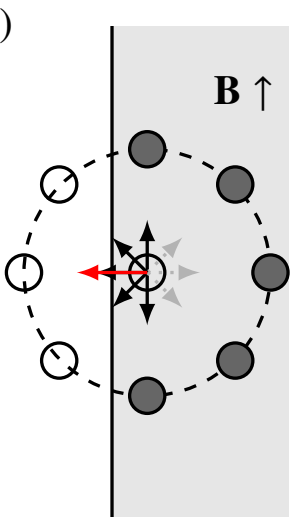

Figure 3. (a) Ferromagnetic dipolar interaction for a vertical magnetic field, either repulsive or attractive depending on the relative position of the particles and the applied magnetic field, quantified by the angle $\alpha$. (b) Forces acting on an off-axis particle. (c) Random close packing generation using DPM simulations. (d) Forces representations inside a tube layer, averaged along $\theta$, for a vertical magnetic field where red (resp. blue) vectors represents positive (resp. negative) radial components.

radial component $f^{r}$. We clearly see that the forces are mainly situated along the tube wall and pushing against it while they rapidly decrease to zero in the packing core. Moreover, we can notice that those radial repulsive forces emerging at the walls of the silos appear constant.

To express the impact of the magnetic forces on the apparent mass at the tube base, we consider the ferromagnetic grain assembly as a whole. Therefore, we no longer consider the local individual forces, but the global net force exerted on the wall $F^{r}=\sum_{i} f_{i}^{r}$. We noticed that the local forces, in the case of a vertical magnetic field, are mainly pushing against the wall ; thus, we expect a positive global radial force. Moreover, if we consider Janssen's hypothesis that the friction forces are fully mobilized in the vertical direction, this global magnetic radial force can provide a global vertical contribution to the pressure measured at the bottom of the silo : $F=-\mu F^{r}$. The final 
(a)

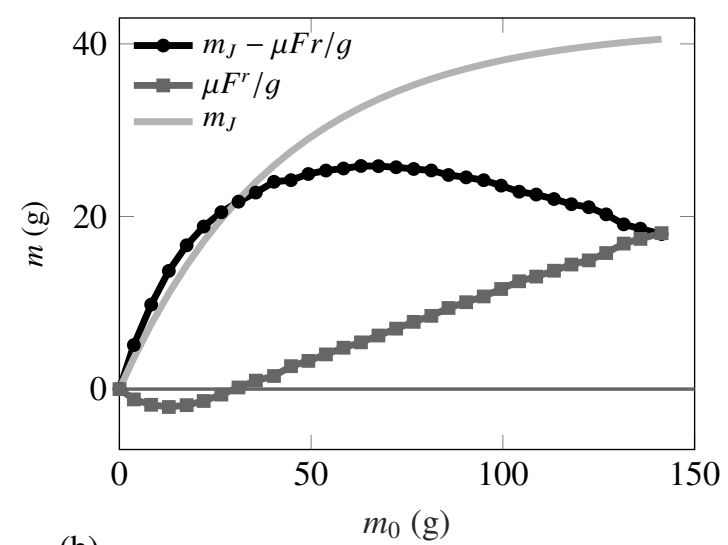

(b)

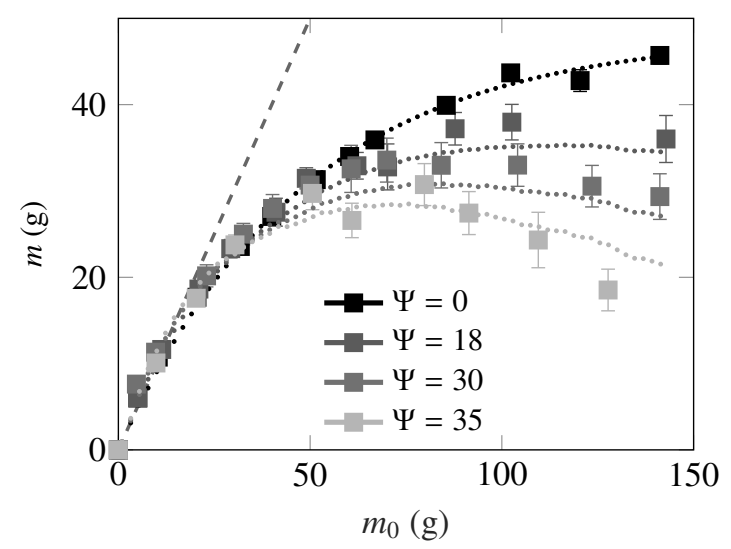

Figure 4. (a) Model curves for a vertical magnetic field. The light grey line shows the classical Janssen prediction; the grey squares line is the result of the computed magnetic mass loss $\mu F^{r} / g$; the black circles line (difference of both curves) gives the predicted apparent mass of the column. (b) Comparison of the model to the experimental data points.

apparent mass is then given by the Janssen approach from which we subtract the magnetic friction contribution:

$$
m=m_{J}-\mu F^{r} / g
$$

where the friction coefficient $\mu$ is equal to 0.4 in our experiments. The global picture of the numerical resolution is given in figure 4 where we show the Janssen contribution $m_{J}$, the global radial force $F^{r}$ computed using the local forces $f_{i}$ for different packing height $h$, and the final result of eq. 2 . The radial force is slightly negative for low mass $m_{0}<30 \mathrm{~g}$ and follow a linear tendency for higher mass. The final shape was compared with the experimental results for different field intensity and was found in good accordance as shown in figure $4 \mathrm{~b}$.

To conclude, we have described a "magnetic Janssen effect" [21], which allows to control the apparent mass of a ferromagnetic granular column. Such ability to finely tune frictional interactions thanks to an external controlling parameter opens appealing perspectives beyond such a static behaviour, with novel dynamical properties involved for instance in the discharge of silos $[15,22]$.

\section{References}

[1] H.A. Janssen, Vereins Eutscher Ingenieure Zeitschrift 39, 1045 (1895)

[2] L. Vanel, E. Clément, Eur. Phys. J. B 11, 525 (1999)

[3] L. Vanel, P. Claudin, J.P. Bouchaud, M.E. Cates, E. Clément, J.P. Wittme, Phys. Rev. Lett. 84, 1439 (2000)

[4] P.G. de Gennes, Rev. Mod. Phys. 71, S374 (1999)

[5] M.E. Cates, J.P. Wittmer, J.P. Bouchaud, P. Claudin, Phys. Rev. Lett. 81, 1841 (1998)

[6] I. Bratberg, K.J. Måløy, A. Hansen, Eur. Phys. J. E 18, 245 (2005)

[7] J.P. Bouchaud, M.E. Cates, P. Claudin, J. Phys-Paris I 5, 639 (1995)

[8] B. Andreotti, Y. Forterre, O. Pouliquen, Granular media: between fluid and solid (Cambridge University Press, Cambridge, 2013), 86

[9] G. Ovarlez, C. Fond, E. Clément, Phys. Rev. E 67, 060302(R) (2003)

[10] J.L. Anthony, C. Marone, J. Geophys. Res. 110, B08409 (2005)

[11] G.W. Baxter, R.P. Behringer, T. Fagert, G.A. Johnson, Phys. Rev. Lett. 62, 2825 (1989)

[12] D.J. Hornbaker, R. Albert, I. Albert, A.L. Barabási, P. Schiffer, Nature 387, 765 (1997)

[13] M. Scheel, R. Seemann, M. Brinkmann, M.D. Michiel, A. Sheppard, B. Breidenbach, S. Herminghaus, Nature Materials 7, 189 (2008)

[14] F. Tapia, S. Santucci, J.C. Géminard, EPL-Europhys. Lett. 115, 64001 (2016)

[15] G. Lumay, N. Vandewalle, Phys. Rev. E 78, 061302 (2008)

[16] J. Vessaire, N. Plihon, R. Volk, M. Bourgoin, Phys. Rev. E 102, 023101 (2020)

[17] K. Bai, J. Casara, A. Nair-Kanneganti, A. Wahl, F. Carle, E. Brown, JPN J. Appl. Phys. 124, 123901 (2018)

[18] B.F. Edwards, J.M. Edwards, Eur. Phys. J 38, 015205 (2016)

[19] T. Weinhart, L. Orefice, M. Post, M.P. van Schrojenstein Lantman, I.F. Denissen, D.R. Tunuguntla, J. Tsang, H. Cheng, M.Y. Shaheen, H. Shi et al., Comput. Phys. Commun. 249, 107129 (2020)

[20] J.W. Landry, G.S. Grest, L.E. Silbert, S.J. Plimpton, Phys. Rev. E 67, 041309 (2003)

[21] L. Thorens, K.J. Måløy, M. Bourgoin, S. Santucci, Nat. Commun. in press (2021)

[22] L. Thorens, K.J. Måløy, M. Bourgoin, S. Santucci, EPJ Web Conf. this volume (2021) 